\title{
Interaction of Wetting Fronts with an Impervious Surface - Longer Time Behaviour
}

\author{
J.-Y. PARLANGE ${ }^{1}$, W. L. HOGARTH ${ }^{2}$, C. FUENTES ${ }^{3}$, J. SPRINTALL ${ }^{4}$, \\ R. HAVERKAMP ${ }^{3}$, D. ELRICK ${ }^{5}$, M. B. PARLANGE ${ }^{6}$, \\ R. D. BRADDOCK ${ }^{2}$, and D. A. LOCKINGTON ${ }^{7}$ \\ ${ }^{1}$ School of Earth Sciences, Flinders University, Adelaide, S.A., Australia. 5000. \\ ${ }^{2}$ Faculty of Environmental Sciences, Griffith University, Nathan, Qld., Australia. 4111. \\ ${ }^{3}$ Institut de Mecanique, Université Joseph Fourier, BP 53-38041 Grenoble-Cedex, France. \\ ${ }^{4}$ Scripps Institution of Oceanography, 9500 Gilman Drive La Jolla, CA. 92093. \\ ${ }^{5}$ Department of Land Resources Science, University of Guelph, Ontario, Canada NIG 2Wl. \\ ${ }^{6}$ Land, Air and Water Resources and Agricultural Engineering, University of California, Davis, CA. \\ 95616. \\ ${ }^{7}$ Department of Civil Engineering, University of Queensland, St. Lucia, Qld. 4072, Australia.
}

(Received: 15 November 1993; in final form: 30 May 1994)

\begin{abstract}
The interaction of a wetting front with an impervious surface can be described very easily in the early stages of interaction by using a superposition principle. After the time when the superposition principle fails to describe the interaction properly, two flow regimes are analysed. For most of the interaction the profile is only affected by the impervious surface near that surface and in particular the inflow into the soil layer is unchanged. Then, only at the very end of the process is the inflow decreasing because of the impervious surface.
\end{abstract}

Key words: Infiltration, layered soils, impervious surface.

\section{Introduction}

In an earlier paper (Parlange et al., 1994), a superposition principle was obtained and its application illustrated by predicting accurately the interaction of a wetting front and a surface. It is only reliable to estimate small corrections and, for the particular problem considered, this means that the early stages of the interaction are accurately described.

In this paper, a method is presented to extend, very simply, the analytical results beyond those early stages. This paper completes the study of the interaction for all times when the principle fails. It also illustrates an important application of the principle which is to facilitate the analysis of a problem by providing the initial response especially when it is singular.

As always, the approximation here is not unique. Rather, of the various methods we tried, we present one which is a compromise between simplicity and accuracy. More importantly, the solution gives us an understanding of the various regimes involved by the interaction of a wetting front and a surface. 
As in Parlange et al. (1994) the results are written for an arbitrary diffusivity. Comparison with an essentially exact numerical simulation is then carried out for an exponential diffusivity,

$$
D=D_{0} \exp n \theta \text {. }
$$

The numerical solution is more reliable for $n$ small and the analytical one for $n$ large. In practice, $n$ is fairly large, around eight (Reichardt et al., 1972), where the present numerical scheme (Braddock et al., 1982; Parlange et al., 1982) does not hold very well. For this reason, the comparison is made for $n=4$, where both can be used. If the analytical result is acceptable for this low value of $n$, then it is more accurate for $n>4$, e.g. 8 .

\section{Theory}

We solve the diffusion equation,

$$
\frac{\partial \theta}{\partial t}=\frac{\partial}{\partial x}\left[D \frac{\partial \theta}{\partial x}\right]
$$

with the initial conditions and boundary conditions,

$$
\begin{aligned}
& \theta=0, \quad t \leqslant 0, \quad 0 \leqslant x \leqslant L, \\
& \partial \theta / \partial x=0, \quad x=L, \\
& \theta=1, \quad x=0, \quad t>0,
\end{aligned}
$$

where $L$ is the depth of the soil layer, $x$ and $t$ are position and time, and $\theta$ is the reduced water content. The superposition principle has provided us with a solution valid for $\theta_{L}$ (value of $\theta$ at $x=L$ ) not too large. We consider the case of a front moving horizontally, i.e., the effect of gravity is not considered. The latter would not effect the qualitative behaviour of the phenomenon which is controlled by the rapid increase of $D$ with $\theta$. Since the interaction is most difficult to describe in the early stages when $\theta_{L}$ increases most rapidly, we expect that, by the time the principle fails, the water profiles and the processes can be described more easily. For this reason, we shall use the simplest approximations to describe the profiles using the double integration technique (Parlange, 1980). At its simplest form, $\partial \theta / \partial t$ is neglected in (2) so that the flux $D \partial \theta / \partial x(\equiv-q)$ is, essentially, independent of $x$. Here this is not possible everywhere because of (4). Thus, instead of taking $q$ constant, we distort it near $x=L$ to satisfy (4). For instance, we can simply take a generalized Taylor expansion near the boundary at $x=L$,

$$
D \partial \theta / \partial x=-q^{*}\left(1-(x / L)^{\nu-1}\right)
$$


which satisfies (4). The coefficient $q^{*}$ in (6) would obviously be the flux $q$ if (6) were exact. In fact, since (6) is a rather crude approximation, $q^{*}$ is also a crude approximation of $q$. It is because $q \neq q^{*}$ that Parlange (1975) introduced an integral method rather than assume $q^{*}=q$, as was done in the original paper of Parlange (1971). Similarly, the value of the parameter $\nu$ entering (6) is unknown a priori.

For any given $D,(6)$ is integrated. For instance, if (1) holds separation of variables leads to,

$$
\theta=\frac{1}{n} \ln \left\{\exp n-\left[\exp n-\exp n \theta_{L}\right] \frac{(x / L)}{\nu-1}\left[\nu-(x / L)^{\nu-1}\right]\right\} .
$$

where $\theta_{L}$ is the value of $\theta$ at $x=L$, i.e., in general by integrating (6) between the two boundaries at $x=0$ and $x=L$,

$$
\int_{\theta_{L}}^{1} D \mathrm{~d} \theta=q^{*} L(\nu-1) / \nu .
$$

which yields $q^{*}$ when $\theta_{L}$ is given.

Even when the superposition principle fails and the predicted $\theta_{L}$ is too large, the cumulative infiltration $I$ is given for a long time by (see Parlange et al., 1994).

$$
I=S \sqrt{t},
$$

as with a semi-infinite medium. The reason is clear: the increase in $\theta_{L}$ is due to interaction between the wetting front and the wall at $x=L$. This will go on for a long time without affecting the water entering the soil at $x=0$ as long as $\theta_{L}$ is sufficiently small. Then the diffusivity at the wall is much smaller than at the sources and diffusion is an inefficient process to transmit the signal from the wall. Thus, from (7), we calculate

$$
I=\int_{0}^{L} \theta \mathrm{d} x
$$

which, from (7) gives $\theta_{L}(t)$ when $\nu$ is given. Figure (1) gives predictions of $\theta_{L}$ for a few $\nu$ 's and $n=4$. A few features are apparent: $\theta_{L}(t)$ does not change drastically with $\nu$, especially as $t$ increases, all curves tend to merge. If we compare with the exact numerical result, the best choice for $\nu$ is about 5 when $\theta_{L} \simeq 0.7$ and about 5.5 for $\theta_{L} \simeq 0.3$. Finally, all curves diverge from the exact results for $D_{0} t / L^{2}>0.035$. This is obviously true because (9) cannot hold for all times. Eventually, the entering water is affected by the wall at $x=L$. Figure (2) gives $I$ obtained numerically as a function of time. It shows that indeed (9) holds as long as $D_{0} t / L^{2}>0.035$.

\section{Determination of $\nu$}

The superposition principle leads to a $\theta_{L}(t)$ reliable for a short time only. We choose $\nu$ by picking the $\theta_{L}(t)$ in Figure 1, which overlaps with, i.e. is tangent 


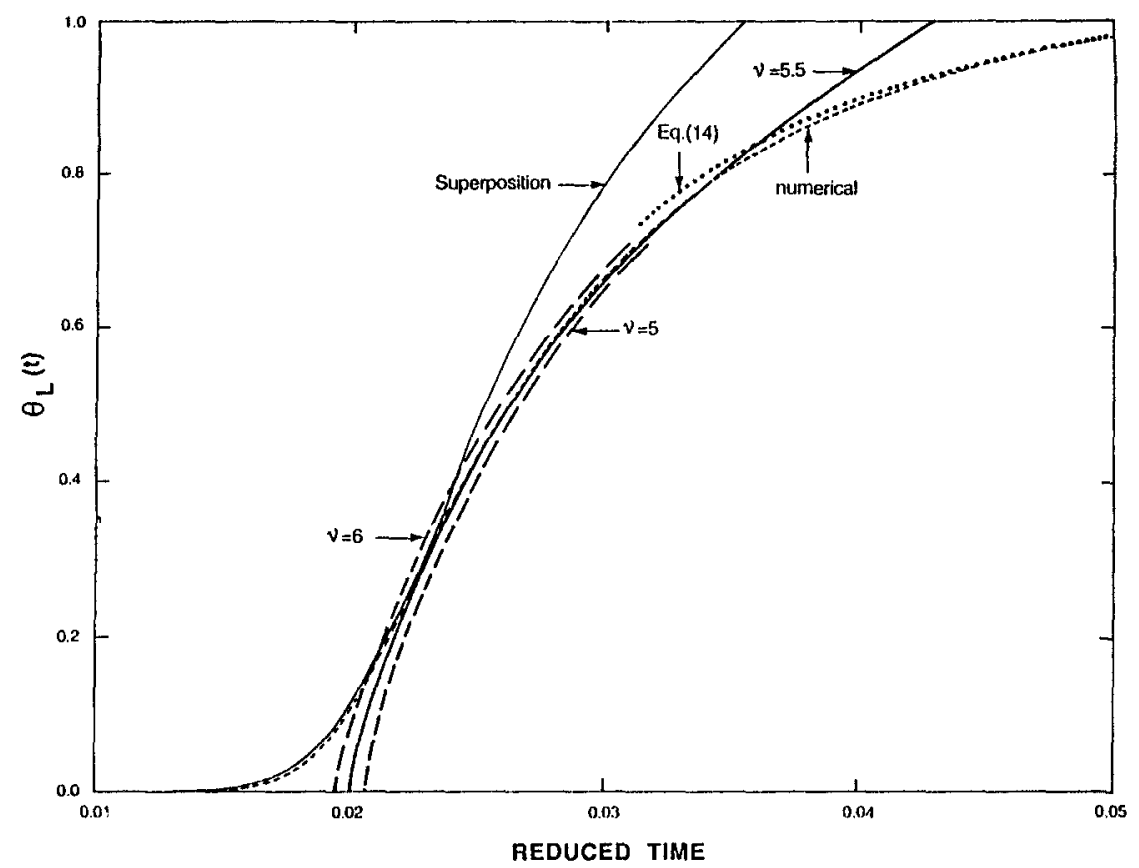

Fig. 1. $\theta_{L}$ as a function of the reduced time $D_{0} t / L^{2}$ : Numerical prediction (thin dashed line) and, result of superposition principle (thin solid line), (7) and (9) with $n=5.5$ (thick solid line), with $n=6$ (left thick dashed line) and $n=5$ (right thick dashed line), (14) with the constant equal to 0.10525 (dotted line).

to, the $\theta_{L}$ obtained by superposition. Here, in the present example, we take, for instance, $\nu=5.5$ (As the influence of $\nu$ is not crucial, the choice does not require an elaborate procedure. We could just as well have taken $\nu=5$, for instance.) Superposition then is used to obtain $\theta_{L}$ for $D_{0} t / L^{2} \leqslant 0.022$ and the present one with $\nu=5.5$ after that time, where the two curves are tangent. This very simple procedure yields $\theta_{L}(t)$, which is obviously a great improvement over $\theta_{L}(t)$ for $D_{0} t / L^{2}>0.022$ obtained by superposition. It is important to notice however that the superposition principle is crucial to estimate $\nu$.

We are now going to improve the prediction when (9) does not describe $I(t)$ accurately. At that time, we shall use the condition obtained by double integration of (2) to obtain $\theta_{L}$, or

$$
\frac{\mathrm{d}}{\mathrm{d} t} \int_{0}^{L} \mathrm{~d} x \int_{x}^{L} \theta \mathrm{d} \bar{x}=\int_{\theta_{L}}^{1} D \mathrm{~d} \theta
$$

In principle, $\theta$ from (7) can be put in (11) to obtain an ordinary differential equation giving $\theta_{L}(t)$. In practice, by the time (9) does not hold, $\theta_{L}>0.8$, so that the profile has little structure and in (11) we replace $\theta$ by the simplest interpolation, i.e. linear,

$$
(1-\theta) /\left(1-\theta_{L}\right)=x / L
$$




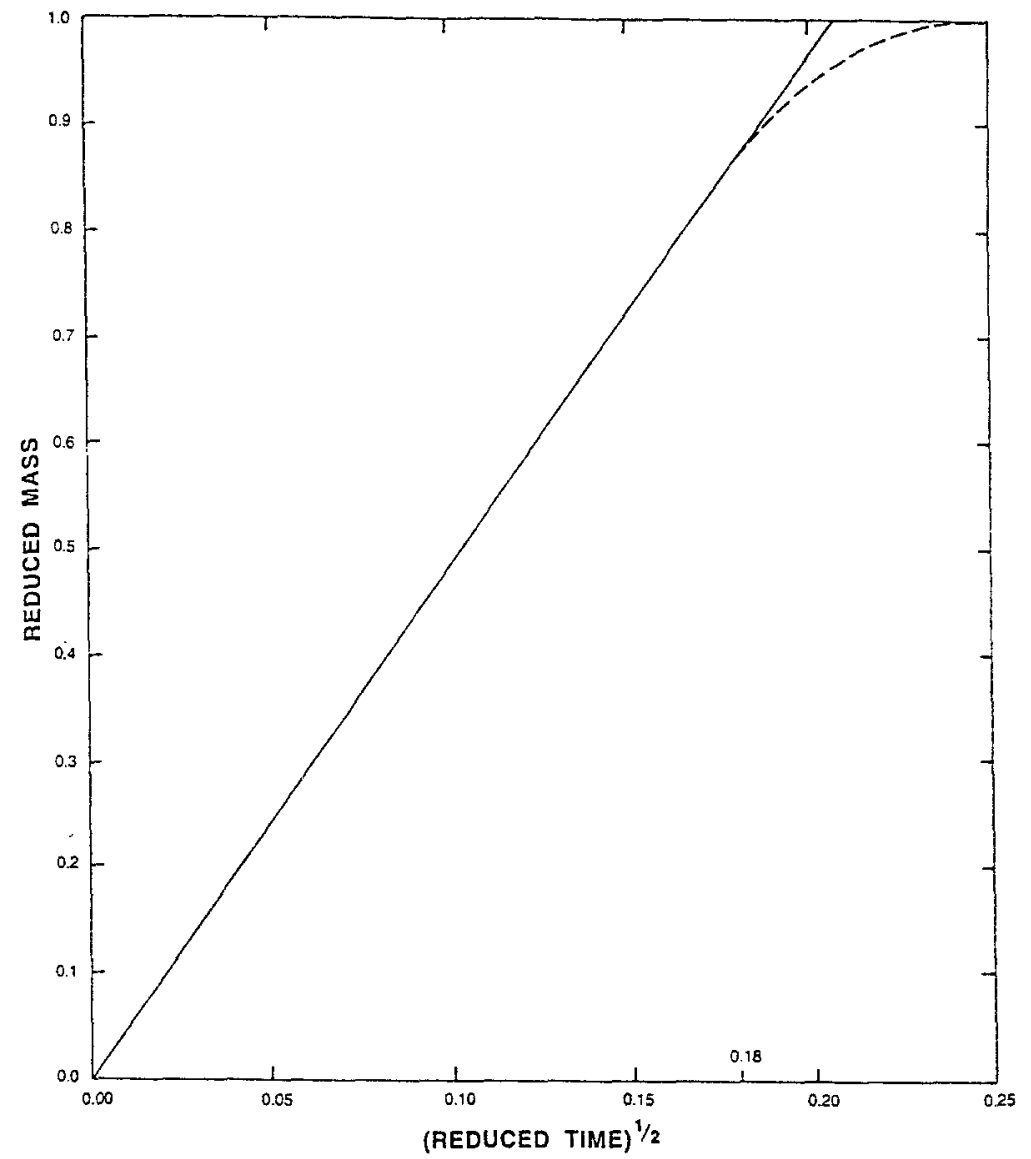

Fig. 2. Cumulative infiltration given as the reduced mass $I / L$, where $I=\int_{0}^{L} \theta \mathrm{d} x$, as a function of the square root of the reduced time $\sqrt{t D_{0} / L^{2}} . S=4.8331 \sqrt{D_{0}}$ and $A=0.1284$.

which is quite accurate enough to put in an integral equation. Then (11) yields, at once, the simple ordinary differential equation

$$
L^{2} \mathrm{~d} \theta_{L} / \mathrm{d} t=3 \int_{\theta_{L}}^{1} D \mathrm{~d} \theta
$$

For instance, in the present example,

$$
3 D_{0} t / L^{2}=\exp -4 \ln \left[\exp 4\left(1-\theta_{L}\right)-1\right]^{-1}+\text { Constant }
$$

the constant of integration being unknown, $\theta_{L}(t)$ is known only up to a translation in time.

We note that the superposition principle yields (9) until $\theta_{L}=1$ at $D_{0} t / L^{2} \simeq$ 0.0353 . Thus it is natural to calculate the constant by matching $\theta_{L}(t)$ given by (14) 


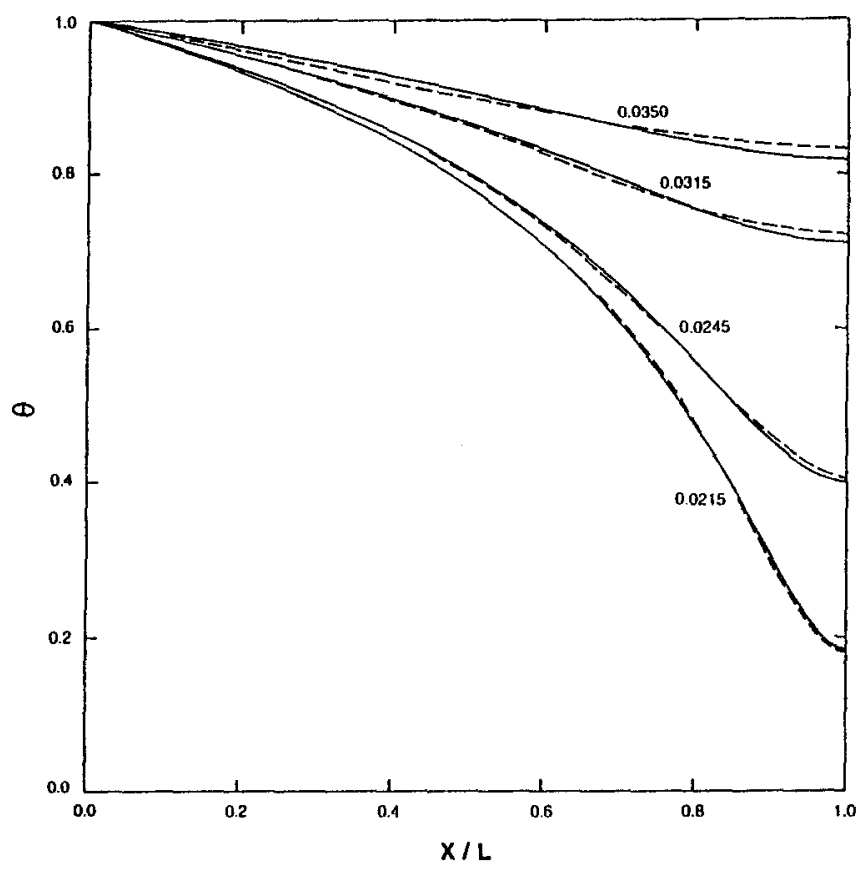

Fig. 3. Profiles calculated from (7) with $n=5.5$ and $\theta_{L}$ given by (9) (dashed line) and numerically (solid line).

with 0.825 given by (7). Thus the constant is found to be equal to 0.10525 . The result is shown on Figure 1 and is remarkably accurate for $\theta_{L}>0.8$.

Knowing $\theta_{L}(t)$, it is easy to use (7) and obtain profiles for all times. Figure 3 gives such profiles for different times. As in Parlange et al. (1994), the accuracy is good, with the worst agreement occurring at $x \simeq L$.

Figure 4 also gives $I$ as a function of $\sqrt{D_{0} t / L^{2}}$ for $D_{0} t / L^{2} \leqslant 0.0353$, when (9) applies and for $D_{0} t / L^{2}>0.353$, when $I$ is calculated from (10), $\theta_{L}(t)$ being known. The agreement is very good, the worst error being at $D_{0} t / L^{2} \simeq 0.0353$, i.e., at that time, the entering flux is already less than $S / 2 \sqrt{D_{0} t / L^{2}}$. However, this minor error is acceptable, considering the simplicity of the approach.

\section{Conclusion}

We have provided an analytical solution to describe the interaction of a wetting front and impervious surface. Comparison with an accurate numerical solution when the soil-water diffusivity does not increase too rapidly with water content, shows the precision of the approach. This is crucial since the approach becomes more accurate as diffusivity increases more rapidly with water content. The simple analysis provides quantitative information on water profiles as a function of time and position, water intake and water content at the interface. 


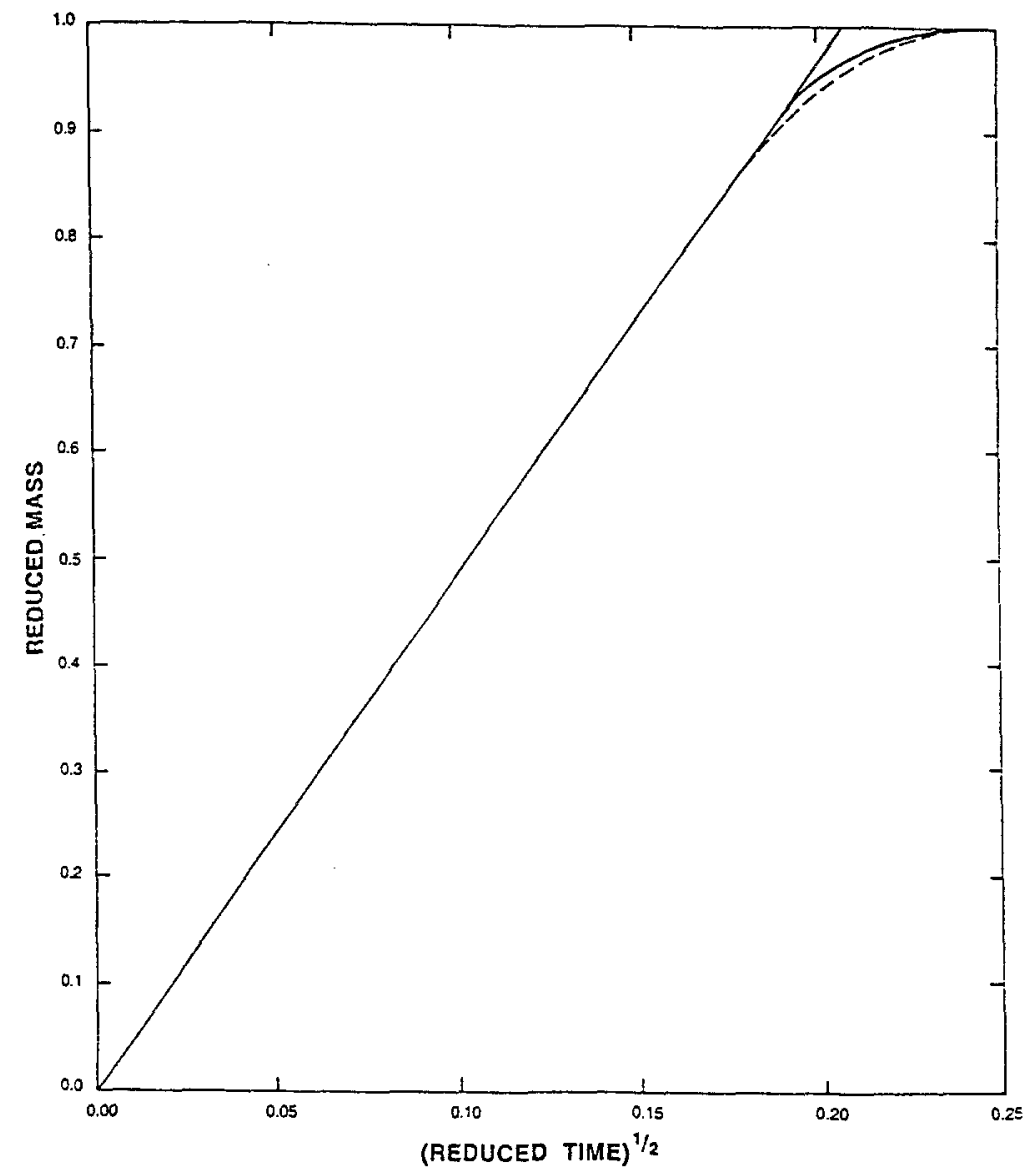

Fig. 4. Reduced mass. Same as Figure 2, with a solid line correction when $\theta_{L}$ given by the dotted line in Figure 1, $\theta$ given by (12) and $I$ given by (10).

More importantly maybe, the analysis gives us a qualitative understanding of the processes involved. At very short times, water infiltrates as in a semi finite medium. Then the wetting front starts interacting with the interface. At the early stages of interaction, a superposition principle is applicable, such that the incoming water is "reflected" by the interface without affecting the water intake. There is an intermediary stage when the water intake remains that for a semi-infinite medium, but the water content at the interface grows more slowly than at the beginning of the interaction. Finally, the water intake itself slows down significantly below that of a semi-infinite medium.

The four regimes have also been quantified by simple analytical formulas. The initial behaviour of the solution obtained in Parlange et al. (1994) by superposition is crucial to obtain a quantitative description of the subsequent regimes when the superposition principle fails. 


\section{References}

Braddock, R. D., Parlange, J. Y., Lockington, D. A. and Doilibi, P., 1982, Nonlinear diffusion with a barrier, in B. J. Noye (Ed.), Numerical Solutions of Partial Differential Equations, pp. 511-525.

Parlange, J. Y., 1971, Theory of water movement in soils: 1. One dimensional absorption. Soil Sci. 111, 134-137.

Parlange, J. Y., 1975, On solving the flow equation in unsaturated soils by optimisation: Horizontal infiltration, Soil Sci. Soc. Am. Proc. 39, 415-418.

Parlange, J. Y., 1980, Water transport in soils, Am. Rev. Fluid Mech. 12, 77-102.

Parlange, J. Y., Hogarth, W. L., Fuentes, C., Sprintall, J., Haverkamp, R., Elrick, D., Parlange, M. B., Braddock, R. D. and Lockington, D. A., 1994, Superposition principle for short-term solutions of Richards' equation - application to the interaction of wetting fronts with an Impervious Surface, Trans. Porous Media 17, 239-247.

Parlange, J. Y., Lockington, D. A. and Braddock, R. D., 1982, Nonlinear diffusion in a finite layer, Bull. Aust. Math. Soc. 26, 249-262.

Reichardt, K., Nielsen, D. R. and Biggar, J. W., 1972, Sealing of horizontal infiltration into homogeneous soils, Soil Sci. Soc. Am. Proc. 36, 241-245. 